\title{
Antiproliferative Activity of Amathaspiramide Alkaloids and Analogs
}

\author{
Jun Shimokawa, ${ }^{a}$ Koji Chiyoda ${ }^{b}$ Hirotatsu Umihara, ${ }^{b}$ and Tohru Fukuyama*a \\ ${ }^{a}$ Graduate School of Pharmaceutical Sciences, Nagoya University; Furo-cho, Chikusa-ku, Nagoya 464-8601, \\ Japan: and ${ }^{b}$ Graduate School of Pharmaceutical Sciences, University of Tokyo; 7-3-1 Hongo, Bunkyo-ku, Tokyo \\ 113-0033, Japan. \\ Received March 14, 2016; accepted March 30, 2016; advance publication released online May 10, 2016
}

\begin{abstract}
Assisted by the total syntheses of all the amathaspiramides, six natural products and four synthetic intermediates with partially fluctuating structures were prepared and subjected to a growth inhibition assay in four human cancer cell lines. The results showed amathaspiramides $\mathrm{A}, \mathrm{C}$, and $\mathrm{E}$ had moderate antiproliferative activity. Examination of the structure-activity relationship revealed the importance of the amine or imine substructure on the pyrrolidine moiety and the $8 R$ stereochemistry on the $N$-acyl hemiaminal moiety for the antiproliferative activity of amathaspiramide alkaloids.
\end{abstract}

Key words amathaspiramide; divergent synthesis; antiproliferative activity; cell viability assay; natural product synthesis

Amathaspiramide alkaloids (1-6) were isolated by Morris and Prinsep in 1999 from a New Zealand collection of marine bryozoan Amathia wilsoni. ${ }^{1)}$ The structural characteristics of these compounds ranges from the highly functionalized diazaspiro[3.3]nonane framework, an intriguingly stable $\mathrm{N}$ acyl hemiaminal, a dibrominated methoxyphenyl group, and a variably oxidized pyrrolidine moiety connected through a C-5 tetrasubstituted spiro center. Bioactivities of these molecules have been reported only partially. Among them, antiviral activity against Poliovirus type 1, antimicrobial activity toward Bacillus subtilis, and cytotoxicity to BSC-1 cells were reported for amathaspiramides A (1) and E (5). ${ }^{1)}$ These $\alpha$-tertalkylamino acids in general are known to be enzyme inhibitor themselves, ${ }^{2)}$ as well as to add conformational twist or rigidity to peptides. ${ }^{3)}$ A recent report also proposed structurally similar compounds as $\beta$-turn mimetics. ${ }^{4)}$ Despite these promising reports, the limited availability of amathaspiramides has hampered further biological investigations on these molecules and their analogs. Of the six natural congeners, Hughes and Trauner, ${ }^{5)}$ Ohfune and colleagues, ${ }^{6,7)}$ and Soheili and Tambar ${ }^{8)}$ reported total synthesis of amathaspiramide $\mathrm{F}$, an only $8 \mathrm{~S}$ member. Eventually, our group has reported the only comprehensive syntheses of all the amathaspiramides, ${ }^{9}$ ) and Lee and colleagues reported the efficient formal synthesis through our common intermediate. ${ }^{10)}$ From the outset of our synthetic study, we envisioned establishing a scalable and versatile synthetic route to all the amathaspiramides whose pyrrolidine moieties range over cyclic imine, secondary or tertiary amine as well as unsubstituted or $N$-methyl $\gamma$-lactam. Therefore, we employed a systematic access for the spirocyclic core and the variable oxidation states of the spiro pyrrolidine units from a late-stage common intermediate, ${ }^{11)}$ which could enable the preparation of the diverse substructure within a uniform core skeleton for structure-activity relationship (SAR) study. The purpose of the current work was to acquire the novel data on the bioactivity of these natural products as well as the information on the SAR, facilitated by the assay of a group of structurally similar molecules bearing the same core structure.

\section{Results and Discussion}

The outline of the synthesis was reported in our previous publication $^{9)}$ and is to be introduced briefly in this manuscript (Chart 1). The synthesis started with the asymmetric hydrogenation of butenolide 7 to give the lactone $\mathbf{8}$. Sequential acylation and alkylation diastereoselectively gave the quaternary intermediate 9. Removal of benzyl group and Curtius rearrangement followed by the acidic hydrolysis afforded a $\gamma$-lactam 10. Bromination under the forced reaction conditions gave the dibromide $\mathbf{1 1}$ and this was converted to the cyclic imide 12 in two steps. Regio-, chemo-, and diastereoselective reduction of the cyclic imide moiety at the 8-position proceeded only by the use of diisobutylaluminum hydride (DIBAL) as the reagent, which represented the total synthesis of amathaspiramide D (4). This molecule could be considered the common precursor to all the other members. Amathaspiramide D (4) was initially treated with Schwartz reagent that mediated the selective reduction of secondary lactam in the presence of the $N$-acyl hemiaminal and tertiary lactam, which gave amathaspiramide E (5). Reductive methylation of 5 gave amathaspiramide $\mathrm{A}$ (1) and simple reduction of imine gave amathaspiramide $\mathrm{C}(\mathbf{3})$. The stereochemistry of the $\mathrm{C} 8 \mathrm{~N}$ acyl hemiaminal moiety was surprisingly stable and did not epimerize during the transformations. This stable stereochemistry was epimerized only under the basic reaction conditions to give amathaspiramide F (6). Finally, amathaspiramide B (2) was synthesized by the protection of hemiaminal of 4 with ethoxyethyl group under acidic conditions and methylation under basic conditions followed by the acidic hydrolysis of the protecting group.

As the potential anti-proliferative activity of these natural products as well as the synthetic intermediates on human carcinoma cells remained virtually unexplored, we were interested in comparing the biological effects of these structurally closely related compounds on human cancer cell lines. The antiproliferative activity was assessed in vitro using a panel of four human cancer cell lines: HCT116 (colon cancer), PC-3 (prostate cancer), MV4-11 (acute myeloid leukemia), MiaPaCa-2 (pancreas cancer). The assays were conducted over three day incubation period at $37^{\circ} \mathrm{C}$ and were run in dupli- 

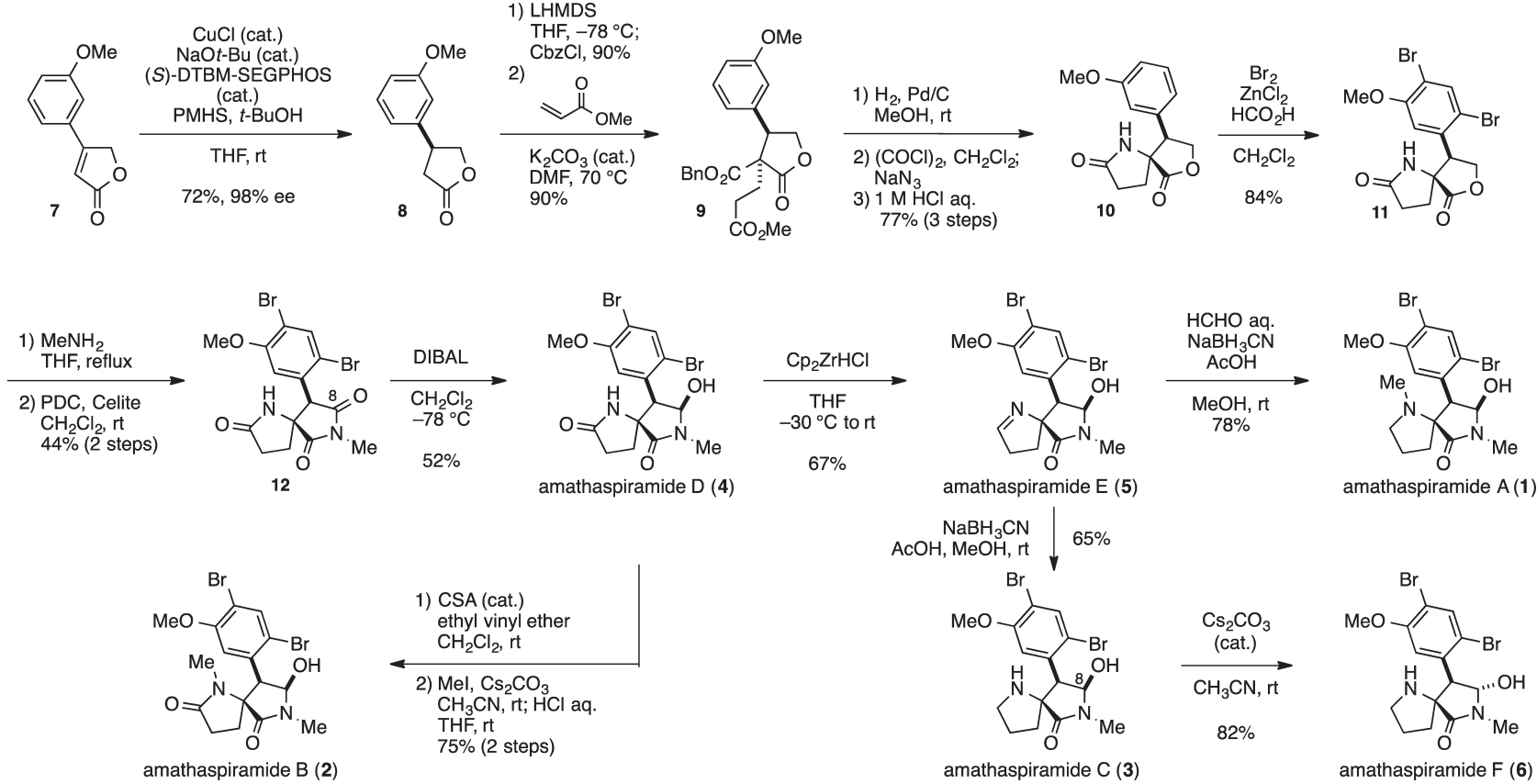

Chart 1. Total Syntheses of All the Amathaspiramides

Table 1. Antiproliferative Effects of Amathaspiramides and Synthetic Intermediates against Human Cancer Cell Lines

\begin{tabular}{|c|c|c|c|c|}
\hline \multirow{2}{*}{ Compound } & \multicolumn{4}{|c|}{$\mathrm{IC}_{50}(\mu \mathrm{M})$} \\
\hline & HCT116 & PC-3 & MV4-11 & MiaPaCa-2 \\
\hline Amathaspiramide A (1) & 46 & 67 & 48 & 14 \\
\hline Amathaspiramide B (2) & $>100$ & $>100$ & $>100$ & $>100$ \\
\hline Amathaspiramide C (3) & 63 & 80 & 64 & 5.8 \\
\hline Amathaspiramide D (4) & $>100$ & $>100$ & 91 & $>100$ \\
\hline Amathaspiramide E (5) & 29 & 81 & 55 & 15 \\
\hline Amathaspiramide F (6) & $>100$ & $>100$ & $>100$ & $>100$ \\
\hline 9 & 64 & $>100$ & $>100$ & 36 \\
\hline 10 & $>100$ & $>100$ & $>100$ & $>100$ \\
\hline 11 & $>100$ & $>100$ & $>100$ & $>100$ \\
\hline 12 & $>100$ & $>100$ & $>100$ & $>100$ \\
\hline Vincristine & - & $<0.015$ & $<0.015$ & - \\
\hline Doxorubicin & - & 0.062 & $<0.013$ & - \\
\hline
\end{tabular}

cate. The results are shown in Table 1. These studies showed that amathaspiramides A (1), C (3), E (5) exhibit moderate antiproliferative activities in all four cell lines, albeit weaker compared to reference standards (vincristine and doxorubicin). Molecules without hemiaminal moiety did not show the strong activity. These results indicated that amathaspiramide alkaloids are confirmed to be the potent antiproliferative agents. SAR between amathaspiramides A (1), C (3), E (5) and B (2), $\mathrm{D}$ (4) indicated that amathaspiramide alkaloids with cyclic amine/imine substructure rather than $\gamma$-lactam were found to be more potent. Also, by comparing the results on amathaspiramides $\mathrm{C}(\mathbf{3})$ and $\mathrm{F}(\mathbf{6})$, the $8 R$ stereochemistry of the $N$-acyl hemiaminal moiety was found to be more potent for the antiproliferative activity.

In conclusion, all the amathaspiramide alkaloids as well as synthetic intermediates were examined for the antiproliferative activity against a panel of four cell lines. Amathaspiramides A (1), C (3), E (5) were found to show the moderate anti- proliferative activity to all four cell lines. With the analysis between the structure and activity, amine or imine moiety in the left-hand pyrrolidine as well as the $8 R$ stereochemistry of the $N$-acyl hemiaminal moiety were indicated to be important for exhibiting antiproliferative activity. These results set the basis for finding the more potent amathaspiramide analogs.

\section{Experimental}

Materials The following human cancer cell lines were obtained from ATC C: HCT116 (ATCC CCL-247), PC-3 (ATCC CRL-1435) MiaPaCa-2 (ATCC CRL-1420), MV4-11 (ATCC CRL-9591).

Determination of Antiproliferative Activity Cells were seeded at density of 2000 cells per well in 96-well plate (Falcon 353075 for HCT116, PC-3 and MiaPaCa-2 cells, Nunclon 163320 for MV4-11 cells) with $100 \mu \mathrm{L}$ of RPMI-1640 medium supplemented with penicillin, streptomycin and 10\% fetal bovine serum (FBS). After overnight culture, cells were treated 
with the medium of appropriate concentration range of compounds $(100 \mu \mathrm{L})$ and cultured for $72 \mathrm{~h}$ at $37^{\circ} \mathrm{C}$ in $5 \% \mathrm{CO}_{2}$. Then $20 \mu \mathrm{L}$ of WST-8 (Cell Counting Kit-8; Dojindo, Kumamoto, Japan) was added to measure viable cells. Absorbance was measured at $450 \mathrm{~nm}$ with Sunrise photometer (Tecan). $\mathrm{IC}_{50}$ values were calculated using percent of growth versus dimethyl sulfoxide (DMSO) treated control.

Acknowledgments The authors thank Dr. Yosuke Kaburagi (Eisai Co., Ltd., Japan) for his support on the growth inhibition assay. This work was supported by JSPS KAKENHI Grant Numbers 23590003, 20002004, 15H05641, 12J10846 and the Platform for Drug Discovery, Informatics, and Structural Life Science from the Ministry of Education, Culture, Sports, Science and Technology of Japan.

Conflict of Interest The authors declare no conflict of interest.

\section{References}

1) Morris B. D., Prinsep M. R., J. Nat. Prod., 62, 688-693 (1999).

2) Pellicciari R., Luneia R., Costantino G., Marinozzi M., Natalini B., Jakobsen P., Kanstrup A., Lombardi G., Moroni F., Thomsen C., J. Med. Chem., 38, 3717-3719 (1995).

3) Maity P., König B., Pept. Sci., 90, 8-27 (2008).

4) Braña M. F., Garranzo M., de Pascual-Teresa B., Pérez-Castells J., Torres M. R., Tetrahedron, 58, 4825-4836 (2002).

5) Hughes C. C., Trauner D., Angew. Chem. Int. Ed., 41, 4556-4559 (2002).

6) Sakaguchi K., Ayabe M., Watanabe Y., Okada T., Kawamura K., Shinada T., Ohfune Y., Org. Lett., 10, 5449-5452 (2008).

7) Sakaguchi K., Ayabe M., Watanabe Y., Okada T., Kawamura K., Shinada T., Ohfune Y., Tetrahedron, 65, 10355-10364 (2009).

8) Soheili A., Tambar U. K., Org. Lett., 15, 5138-5141 (2013).

9) Chiyoda K., Shimokawa J., Fukuyama T., Angew. Chem. Int. Ed., 51, 2505-2508 (2012).

10) O’Connor M., Sun C., Lee D., Angew. Chem. Int. Ed., 54, $9963-$ 9966 (2015)

11) Shimokawa J., Tetrahedron Lett., 55, 6156-6162 (2014). 